\title{
The hydrothermal wave of large-Prandtl- number fluid in a shallow cavity
}

\author{
TANG ZeMei \& HU WenRui \\ National Microgravity Laboratory, Institute of Mechanics, Chinese Academy of Sciences, Beijing 100080, \\ China \\ The hydrothermal wave was investigated numerically for large-Prandtl-number fluid \\ $(P r=105.6)$ in a shallow cavity with different heated sidewalls. The traveling wave \\ appears and propagates in the direction opposite to the surface flow (upstream) in \\ the case of zero gravity when the applied temperature difference grows and over \\ the critical value. The phase relationships of the disturbed velocity, temperature \\ and pressure demonstrate that the traveling wave is driven by the disturbed tem- \\ perature, which is named hydrothermal wave. The hydrothermal wave is so weak \\ that the oscillatory flow field and temperature distribution can hardly be observed \\ in the liquid layer. The exciting mechanism of hydrothermal wave is analyzed and \\ discussed in the present paper.
}

hydrothermal wave, numerical simulation, microgravity

\section{Introduction}

During the development of manned spaceflight and space science, the thermocapillary convection and its instability have attracted much attention in the past decades. It is important in both the basic research of microgravity fluid physics and the applications of materials processing. In 1978, both Schwabe \& Scharmann ${ }^{[1]}$ and Chun $\&$ Wuest ${ }^{[2]}$ observed the oscillatory thermocapillary convection in a floating half zone. The theoretical analyses of thermocapillary instabilities in the liquid layers were given in Pearson's work ${ }^{[3]}$, who pointed out that the Marangoni convection cells appear in a static liquid layer heated from below, when the temperature gradient perpendicular to the layer is sufficiently large although without gravity. Smith and Davis ${ }^{[4]}$ suggested that, in a general situation, the imposed temperature gradient has a component parallel to the free surface, which drives the convection in the liquid layer. They studied the linear stability of a liquid layer with infinite horizontal extension in case of zero gravity and the basic state is not a static one, and found two types of thermal-convective instabilities: Stationary longitudinal rolls and unsteady hydro-

Received September 20, 2006; accepted November 27, 2006; published online September 29, 2007 doi: 10.1007/s11433-007-0060-8

†Corresponding author (email: zmtang@imech.ac.cn)

Supported by the National Natural Science Foundation of China (Grant No. 10432060) 
thermal wave. The hydrothermal waves derive their energy from the vertical temperature gradient in the case of large Prandtl fluid, and propagate obliquely in the direction of the surface flow. They believed that the mechanism which induces the hydrothermal waves in an infinite liquid layer should produce the same instability effect in a cylindrical liquid bridge.

In the terrestrial gravity environment the convection and its instability are complex in a liquid layer due to the coupling of the thermocapillary effect and the buoyancy effect, depending on the dynamic Bond number. Burguete et al. ${ }^{[5]}$ reported the experiments on buoyancy-thermocapillary instabilities in the liquid layer $(P r=10)$ subjected to a horizontal temperature gradient. The oblique traveling waves or longitudinal stationary rolls were observed for small and large aspect ratios of the liquid layer. They summarized the results demonstrated in numerous experiments and noticed that only few of the experiments have provided direct evidence of hydrothermal waves. The stationary multi-cellular flows were observed before appearance of the oscillatory convection in many experiments. A 2D numerical simulation and experiments of coupled buoyancy and thermocapillary convection (for acetone, $P r=4$ ) were reported by Villers and Platten ${ }^{[6]}$. They have shown the existence of a multicellular steady flow prior to the oscillatory multicellular convection. Our calculated results on thermocapillary-buoyancy convection for the liquid layer agree with the ones of Villers and Platten for the same parameters.

Thermocapillary-buoyancy convection in a shallow cavity heated from the sides was investigated numerically by Shevtsova et al. ${ }^{[7]}$. Their results for Prandtl number 13.9 with an aspect ratio $A=24.7$ showed that the unicellular flow is replaced by a steady bicellular or multicellular flow during the increase of Marangoni number $M a$, and then either a hydrothermal wave or an oscillatory multicellular flow appears during the further increase of $M a$, depending on the dynamic Bond number. They considered that the oscillatory multicellular flow is induced by the disturbance generated near the hot wall. The dependence of critical Marangoni number from a steady state to an oscillatory state was given in the region of the dynamic Bond number $0-0.8$ in their transition map.

The large-Prandtl-number liquid layer, for example $10 \mathrm{cSt}$ silicon oil $(\mathrm{Pr}=105.6)$, has larger viscosity and lower conductivity, and then may have different behavior of oscillatory convection from that of the fluids with $\operatorname{Pr}<20$, which were usually used in the experiments and numerical simulations. Smith and Davis ${ }^{[4]}$ pointed out that the direction of propagation of the disturbances is only $7.9^{\circ}$ related to the flow direction in the case of $P r=\infty$, therefore the two-dimensional model is a reasonable approximation for studying the large-Prandtl-number fluid layer. The large- $P r$-number liquid is easy for observation in the experimental process, also is relatively stable and not easily influenced by the disturbance. In the present study, we examine the thermocapillary convection and instability of $10 \mathrm{cSt}$ silicon oil in a shallow slot in the microgravity environment by 2D numerical method. The studies focus on analyzing the phase relationship of the disturbed velocity, temperature and pressure for understanding the mechanism exciting hydrothermal wave and for preparing a space experiment.

\section{Physical model and mathematical formulation}

The rectangular cavity was filled with $10 \mathrm{cSt}$ silicon oil $(\operatorname{Pr}=105.6)$. The liquid layer was $4 \mathrm{~mm}$ in height and $52 \mathrm{~mm}$ in length. The temperature $T_{\mathrm{h}}$ at the left side of the cavity was higher than $T_{\mathrm{c}}$ at the right side, and $\Delta T=T_{\mathrm{h}}-T_{\mathrm{c}}$. The bottom boundary was a rigid solid, while the top was a de- 
formable one with a passive gas. Both the bottom and top boundaries are assumed to be thermally isolated. The liquid is considered as incompressible with constant viscosity and thermal diffusivity. The surface tension $\sigma$ on the free surface is a linear function of the temperature $T$, and $-\partial \sigma / \partial T$ is assumed to be constant. The height of the liquid layer $h$, the temperature difference $\Delta T$ and velocity $U_{r}=|\partial \sigma / \partial T| \Delta T / \mu$ are used as the typical units to scale the length, temperature and velocity. The typical pressure is $\mu U_{r} / h$, where $\mu$ is dynamic viscosity. Figure 1 is a sketch of the liquid layer.

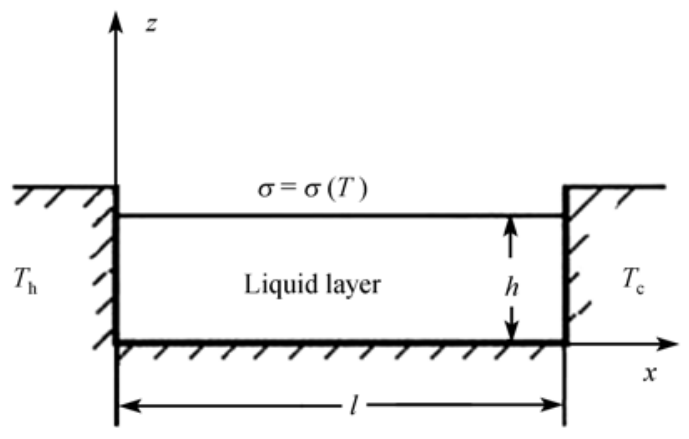

Figure 1 A sketch of liquid layer.

The non-dimensional vorticity $\omega=\left(\omega_{\xi}, \omega_{\theta}, \omega_{\varsigma}\right)$ and stream function $\psi=\left(\psi_{\xi}, \psi_{\theta}, \psi_{\varsigma}\right)$ are introduced, which are defined as

$$
\begin{gathered}
\nabla \times \boldsymbol{\psi}=\boldsymbol{V}, \\
\nabla \times \nabla \times \boldsymbol{\psi}=\boldsymbol{\omega} .
\end{gathered}
$$

The non-dimensional equations and boundary conditions can be expressed by using vorticity $\omega$ and stream function $\psi$. The continuity equation is satisfied automatically. The momentum equation and thermal equation can be expressed respectively as

$$
\begin{gathered}
\frac{\partial \boldsymbol{\omega}}{\partial \tau}+\boldsymbol{V} \cdot \nabla \boldsymbol{\omega}-\boldsymbol{\omega} \cdot \nabla \boldsymbol{V}=\frac{\mathbf{1}}{\operatorname{Re}^{*}} \nabla^{2} \boldsymbol{\omega}, \\
\frac{\partial \Theta}{\partial \tau}+\boldsymbol{V} \cdot \nabla \Theta=\frac{1}{M a^{*}} \nabla^{2} \Theta .
\end{gathered}
$$

For the two-dimensional problem, the boundary conditions are as follows:

$$
\begin{gathered}
\xi=0: \psi_{\theta}=0, \quad \omega_{\theta}=-\frac{\partial W}{\partial \xi}, \Theta(0, \tau)=f(\tau), \\
\xi=1: \psi_{\theta}=0, \quad \omega_{\theta}=-\frac{\partial W}{\partial \xi}, \Theta(1)=0, \\
\varsigma=0: \psi_{\theta}=0, \quad \omega_{\theta}=\frac{\partial U}{\partial \varsigma}, \frac{\partial \Theta}{\partial n}=0, \\
\varsigma=R(\xi, \tau): \psi=0 .
\end{gathered}
$$

The vorticity on the free surface is determined from the equilibrium of tangential stress:

$$
\omega=\frac{\left(1+R^{\prime 2}\right)}{\left(1-R^{\prime 2}\right)} \frac{\partial \Theta}{\partial S}+\frac{2 R^{\prime}}{\left(1-R^{\prime 2}\right)}\left[\frac{\partial}{\partial \xi}\left(-\frac{\partial \psi}{\partial \zeta}\right)-\frac{\partial}{\partial \zeta}\left(\frac{1}{\xi} \frac{\partial \xi \psi}{\partial \xi}\right)\right]+2 \frac{\partial}{\partial \zeta}\left(-\frac{\partial \psi}{\partial \zeta}\right),
$$




$$
\varsigma=R(\xi, \tau): \frac{\partial \Theta}{\partial n}=0 .
$$

And the equilibrium of normal stress is as follows:

$$
\left(P-P_{\infty}\right)=\frac{2}{\left(1+R^{\prime 2}\right)}\left[R^{\prime 2} \frac{\partial U}{\partial \xi}-R^{\prime}\left(\frac{\partial W}{\partial \xi}+\frac{\partial U}{\partial \varsigma}\right)+\frac{\partial W}{\partial \varsigma}\right]-\frac{R^{\prime \prime}}{C a \cdot\left(1+R^{\prime 2}\right)^{3 / 2}},
$$

where $U$ and $W$ are respectively the non-dimensional velocities in the directions $\xi$ and $\zeta$, where $\xi=$ $x / h$ and $\zeta=z / h$. The free surface is described as $\zeta=R(\xi, \tau)$. The free surface at both the left and right boundaries is located at $\zeta=1$, which means that the method of controlling the height of the liquid layer at the interface of solid and liquid is adopted. Here, $n$ is the unit vector in the normal direction of the free surface. The initial condition may be obtained from the static case without the applied temperature difference and convection.

\section{Numerical method and the validation of the program}

Eqs. (3) and (4) with the initial and boundary conditions are solved by using the hybrid finite element method of fractional steps. The numbers of the cell are $121 \times 21$ in the directions $x$ and $z$, respectively, and the calculated domain is divided into 4800 triangular elements with 2541 nodes.

A plane is adopted as the initial free surface and the temperatures at two vertical isothermal side walls are kept at the temperature $T_{0}$ at $t=0$. The temperature at the place $x=0$ is increased from $T_{0}$ to an expected temperature, for example $T_{0}+25^{\circ} \mathrm{C}$, with a heating rate of $0.5^{\circ} \mathrm{C} / \mathrm{s}$, and then the temperature is kept constant. The temperature at the right wall is kept at $T_{0}$. The flow field and temperature distribution in the liquid layer can be obtained numerically. Integrating the tangential pressure gradient along the free surface, obtained from the momentum equations expressed in velocities, and keeping the conservative volume of the liquid layer, the pressure distribution is obtained on the free surface. The configuration of free surface coupled with the flow field and temperature distribution can be obtained from the equilibrium condition of normal stress (11).

The present program is used to calculate the thermocapillary convection in a square container for comparison with the results obtained by Carpenter and Homsy ${ }^{[8]}$. The results are shown in Table 1 , where $-\psi_{\min }$ is the minimum of stream function in the region, and $\omega_{\text {core }}$ is the vorticity at point

\begin{tabular}{|c|c|c|c|c|c|c|c|c|c|}
\hline & Grid & $R e$ & $-\psi_{\min }$ & $\omega_{\text {core }}$ & $x_{\mathrm{c}}$ & $z_{\mathrm{c}}$ & $u(0.5,1)$ & $N u_{x=0}$ & $N u_{x=1}$ \\
\hline \multicolumn{10}{|c|}{$P r=1$} \\
\hline A & $64 \times 64$ & $1.0 \times 10^{4}$ & $3.23 \times 10^{-3}$ & $-6.97 \times 10^{-2}$ & 0.58 & 0.62 & $2.96 \times 10^{-2}$ & 4.36 & 4.40 \\
\hline B & $64 \times 64$ & $1.0 \times 10^{4}$ & $3.20 \times 10^{-3}$ & $-7.10 \times 10^{-2}$ & 0.58 & 0.63 & $2.95 \times 10^{-2}$ & 4.34 & 4.32 \\
\hline \multicolumn{10}{|c|}{$\operatorname{Pr}=10$} \\
\hline A & $64 \times 64$ & $1.0 \times 10^{3}$ & $2.60 \times 10^{-3}$ & $-10.0 \times 10^{-2}$ & - & - & $2.30 \times 10^{-2}$ & 3.79 & 3.83 \\
\hline B & $64 \times 64$ & $1.0 \times 10^{3}$ & $2.75 \times 10^{-3}$ & $-9.56 \times 10^{-2}$ & - & - & $2.47 \times 10^{-2}$ & 3.97 & 3.86 \\
\hline \multicolumn{10}{|c|}{$\operatorname{Pr}=30$} \\
\hline A & $64 \times 64$ & $0.25 \times 10^{3}$ & $2.18 \times 10^{-3}$ & $-9.96 \times 10^{-2}$ & - & - & $1.78 \times 10^{-2}$ & 3.34 & 3.37 \\
\hline B & $64 \times 64$ & $0.25 \times 10^{3}$ & $2.15 \times 10^{-3}$ & $-9.33 \times 10^{-2}$ & - & - & $1.74 \times 10^{-2}$ & 3.51 & 3.41 \\
\hline \multicolumn{10}{|c|}{$\operatorname{Pr}=50$} \\
\hline A & $74 \times 74$ & $0.20 \times 10^{3}$ & $1.96 \times 10^{-3}$ & $-9.07 \times 10^{-2}$ & - & - & $1.53 \times 10^{-2}$ & 3.59 & 3.57 \\
\hline$B$ & $64 \times 64$ & $0.20 \times 10^{3}$ & $2.09 \times 10^{-3}$ & $-9.13 \times 10^{-2}$ & - & - & $1.74 \times 10^{-2}$ & 3.81 & 3.62 \\
\hline$B$ & $74 \times 74$ & $0.20 \times 10^{3}$ & $2.12 \times 10^{-3}$ & $-9.03 \times 10^{-2}$ & - & - & $1.72 \times 10^{-2}$ & 3.84 & 3.64 \\
\hline
\end{tabular}

Table 1 Comparison of Carpenter's results ${ }^{[8]}$ and present results ${ }^{\mathrm{a})}$

a) A, results of Carpenter and Homsy; B, results of the present paper. 
$\left(\xi_{\mathrm{c}}, \zeta_{\mathrm{c}}\right)$. The non-dimensional velocity at $\xi=0.5$ and $\zeta=1$ is denoted by $u(0.5,1)$. Nusselt number is defined as $N u(\zeta)=\int_{0}^{1}\left(M a \frac{\partial \psi}{\partial \zeta} \Theta-\frac{\partial \Theta}{\partial \xi}\right) \mathrm{d} \zeta$. The results obtained by the hybrid finite element method of fractional steps are coincident with those given by Carpenter. The similar results calculated by two kinds of grids are given in Table 1 for the fluid of $\operatorname{Pr}=50$.

The time-dependent temporary velocity $u$, temperature $T$, stream function $\psi$ and pressure $P$ are divided into two parts, for example $u=u_{0}(x, z)+u^{\prime}(x, z, t)$, where $u_{0}$ is a time-average and steady velocity. The oscillatory component $u^{\prime}$ is obtained from $u-u_{0}$. The disturbance almost expands to the whole liquid layer at about $\Delta T=25^{\circ} \mathrm{C}$. The calculated result for $\Delta T=25^{\circ} \mathrm{C}$ is considered as the typical example for detailed discussion. The non-dimensional average velocity $U_{0}$ along the height $\zeta$ at $\xi=0.5$ in case of $g=0$ and $\Delta T=25^{\circ} \mathrm{C}$ is compared with the basic state of return flow, where $U_{0} t=\frac{3}{4} \varsigma^{2}-\frac{1}{2} \varsigma$ was adopted by Smith and Davis. Table 2 and Figure 2 indicate that the present result coincides very well with that of Smith and Davis. All of the results provide validation for the present program.

Table 2 The distribution of non-dimensional velocity $U_{0}$ along the height $\zeta$ at $\xi=0.5^{\text {a) }}$

\begin{tabular}{cccccccc}
\hline$\zeta$ & 1.0 & 0.8 & 0.6 & 0.4 & 0.2 & 0.0 \\
\hline$U_{0} t$ & 0.250 & 0.080 & 0.030 & -0.080 & -0.070 & 0.000 \\
$U_{0} p$ & 0.248 & 0.081 & 0.029 & -0.079 & -0.0694 & 0.000 \\
\hline
\end{tabular}

a) $U_{0} t$, the results of Smith and Davis, where $U t=\frac{3}{4} \varsigma^{2}-\frac{1}{2} \varsigma ; U_{0} p$, the present results.

\section{Numerical results}

When the temperature difference is larger than zero but lower than the critical value, the fluid near the free surface is driven by the surface tension gradient and flows from the hot side to cold side, while the fluid moves from the cold side to hot side in the region near the bottom of the cavity. The convection is steady. The flow field and temperature distribution are shown in Figure 3, and the temperature gradient along $z$-axis is positive.

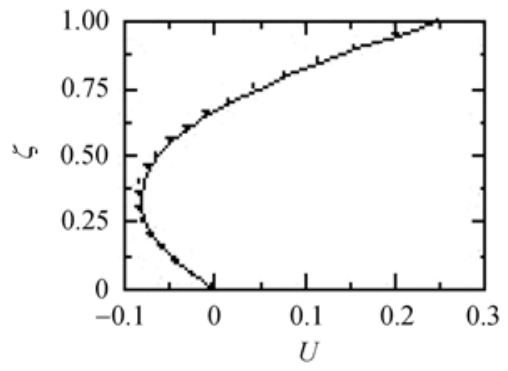

Figure 2 The non-dimensional velocity $U_{0}$ along the height $\zeta$ at $\xi=0.5\left(g=0, \Delta T=25^{\circ} \mathrm{C}\right)$. - , The present result; $\cdot$, the result of Smith and Davis.
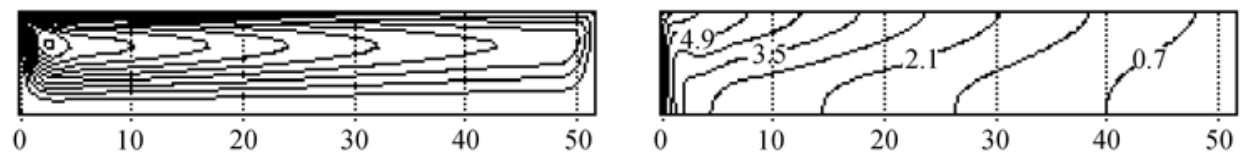

Figure 3 The steady flow field and temperature distribution $\left(g=0, \Delta T=7^{\circ} \mathrm{C}\right)$.

A disturbed cell emerges at first in the region near the hot side when the temperature difference $\Delta T$ is larger than $9^{\circ} \mathrm{C}$. The disturbed cells are distributed in the whole liquid layer when increasing the temperature difference continuously up to $\Delta T=25^{\circ} \mathrm{C}$, and the corresponding temperature difference is defined as the critical temperature difference $\Delta T_{\mathrm{c}}$. Although the disturbed velocity $u^{\prime}$ is 
oscillatory obviously, it is very small so that it is difficult to determine the critical temperature difference from the direct observation of the flow field and temperature distribution in the liquid layer especially in the case of $g=0$. The oscillatory convection can be detected from the spectra of disturbed velocity $u^{\prime}$ at three typical points on the free surface, for example, at $x_{1}=8.233 \mathrm{~mm}, x_{2}=$ $21.233 \mathrm{~mm}$ and $x_{3}=34.233 \mathrm{~mm}$. Figure 4 indicates the spectra of the disturbed velocity $u^{\prime}$ at $x_{1}, x_{2}$ and $x_{3}$ when $\Delta T=25^{\circ} \mathrm{C}$. The oscillatory frequencies of the disturbed velocities $u^{\prime}$ are $0.045144 \mathrm{~Hz}$. The power spectrum $A$ in Figure 4 at $x_{2}$ and $x_{3}$ is stronger than that at $x_{1}$.
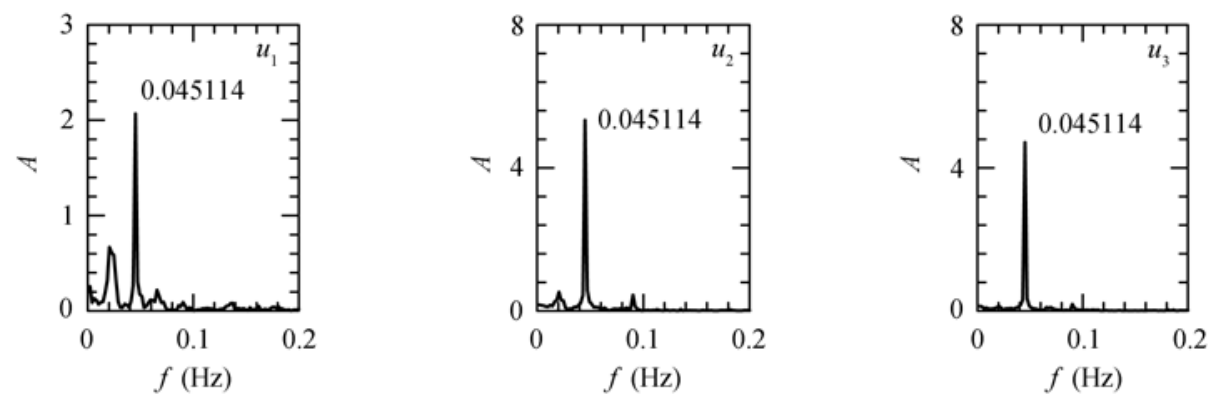

Figure 4 The power spectrum $A$ of the disturbed velocity $u^{\prime}$ at the three positions $x_{1}, x_{2}$ and $x_{3}$ on the free surface $(g=0, \Delta T=$ $\left.25^{\circ} \mathrm{C}\right)$.

The average velocity $u_{0}$, temperature $T_{0}$ and pressure $P_{0}$ on the free surface in the case of $g=0$ and $\Delta T=25^{\circ} \mathrm{C}$ are shown in Figure 5. Different from the infinite-liquid-layer model used in the linear stability analysis, the velocity and temperature distribution on the free surface are not uniform (Figure 5) due to the effect of the side walls. It is shown that $T_{0}$ is distributed linearly in the region between $x=10.4 \mathrm{~mm}$ and $x=40.3 \mathrm{~mm}$. The comparison of the results calculated in this region with that of Smith and Davis shows that the dimensionless wave number and phase velocity are 2.417 and 0.0666 in the present calculation, which are close to the results of 0.47 and 0.0622 obtained by using the linear stability analysis by Smith and Davis for $\operatorname{Pr} \rightarrow \infty$. The oscillatory components of velocity $u^{\prime}$ (Figure 6), temperature $T^{\prime}$ (Figure 7), pressure $p^{\prime}$ (Figure 8) and surface deformation $h^{\prime}$ (Figure 9) on the free surface show the traveling wave propagating in the direction opposite to the velocity on the free surface, called the upstream traveling wave. The magnitude of related disturbance can be seen from Figures (6)-(9).
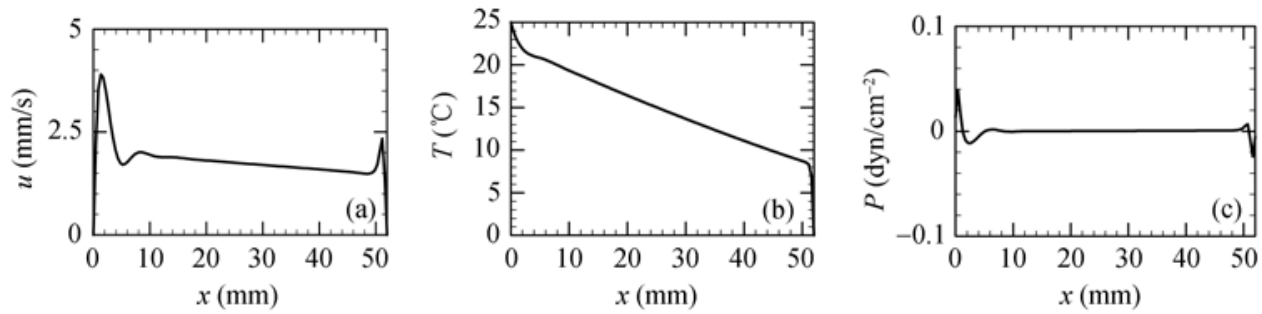

Figure 5 The velocity $u_{0}$ (a), temperature $T_{0}$ (b) and pressure $P_{0}$ (c) on the free surface $\left(g=0, \Delta T=25^{\circ} \mathrm{C}\right)$.

The steady stream function $\psi_{0}$ and temperature $T_{0}$, obtained by averaging the stream function $\psi$ and temperature $T$ in two oscillatory periods, in the case of $g=0$ and $\Delta T=25^{\circ} \mathrm{C}$, are shown in Figure 10. Two steady cells emerge in the region near the hot side in Figure 10(a). The typical temperature distribution of thermocapillary convection in the case of large-Prandtl-number fluid 


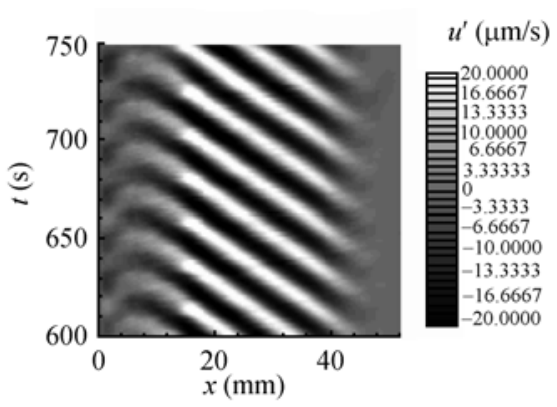

Figure 6 The evolution of velocity $u^{\prime}$ on the free surface ( $g=$ $\left.0, \Delta T=25^{\circ} \mathrm{C}\right)$.

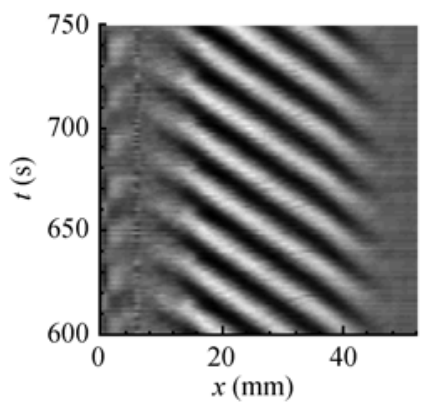

Figure 8 The evolution of pressure $p^{\prime}$ on the free surface ( $g$ $=0, \Delta T=25^{\circ} \mathrm{C}$ ).

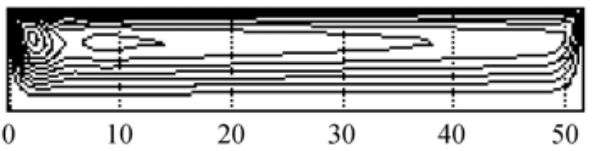

(a)

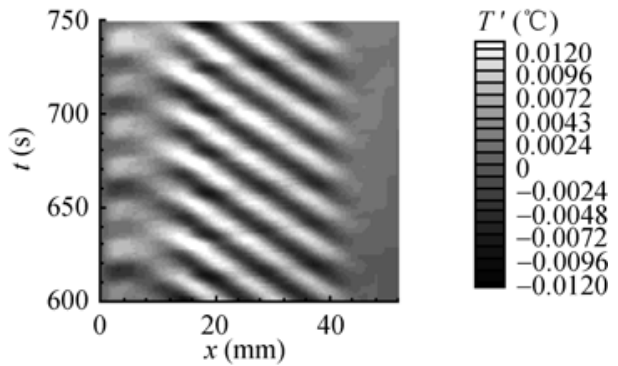

Figure 7 The evolution of temperature $T^{\prime}$ on the free surface $\left(g=0, \Delta T=25^{\circ} \mathrm{C}\right)$.

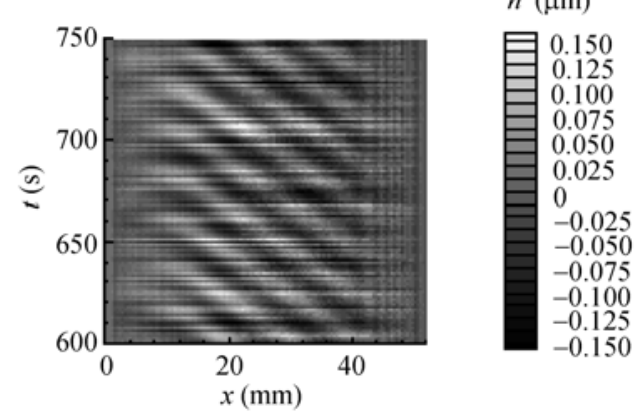

Figure 9 The evolution of surface deformation free surface $\left(g=0, \Delta T=25^{\circ} \mathrm{C}\right)$.

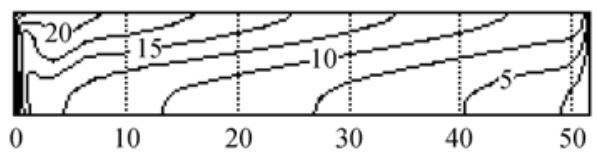

(b)

Figure 10 Average stream function (left) and isotherms in the liquid layer (right). $g=0, \Delta T=25^{\circ} \mathrm{C}$.

layer is shown in Figure 10(b). The temperature gradient is positive along $z$-axis.

The disturbed stream function $\psi^{\prime}$ (left) and disturbed temperature $T^{\prime}$ (right) in the liquid layer are shown in Figure 11, in which the solid and dashed lines correspond, respectively, to the positive and negative values of $\psi^{\prime}$ or $T^{\prime}$. The cells with negative values of stream line (dashed-line) rotate in clockwise, and the solid-line cells rotate in counterclockwise. The disturbed cells originate roughly in the region $x=40-45 \mathrm{~mm}$ and migrate in the direction opposite to the surface flow during the time evolution, then move down in the region near the hot lateral wall. The centers of flow cells are located at the line parallel to $x$-axis at $z=\frac{2}{3} h$. The disturbed temperature pattern is formed due to the effects of average and disturbed velocity, as shown in Figure 11 (right). It is of interest to note that no oscillatory multicelluar flow appears in Figure 12. The patterns of the temporary stream function $\psi$ and temperature $T$ in the liquid layer look like the stationary ones (Figure 12), although the periodic disturbances of flow and temperature do obviously exist (Figure 11). The average fields of the stream function and temperature distribution shown in Figure 10 are similar to the related temporary fields in Figure 12. The magnitudes of $u^{\prime}, T^{\prime}$ and $P^{\prime}$ are shown in Table 3. The ratios $\frac{u_{\max }^{\prime}}{u_{0 \max }}, \frac{T_{\max }^{\prime}}{\Delta T}$ and $\frac{P_{\max }^{\prime}}{P_{0 \max }}$ are $0.51 \%, 0.08 \%$ and $0.38 \%$, respectively, which are so small, 
and the multicellular oscillatory flow can hardly be observed obviously from the patterns of temporary stream function $\psi$ and temperature $T$, even if the applied temperature difference grows up to $\Delta T=45^{\circ} \mathrm{C}$. In Table $3, u_{0 \max }, \Delta T$ and $P_{0 \max }$ correspond to the maximum average velocity, the applied temperature difference, and the maximum average pressure on the free surface of the liquid layer.
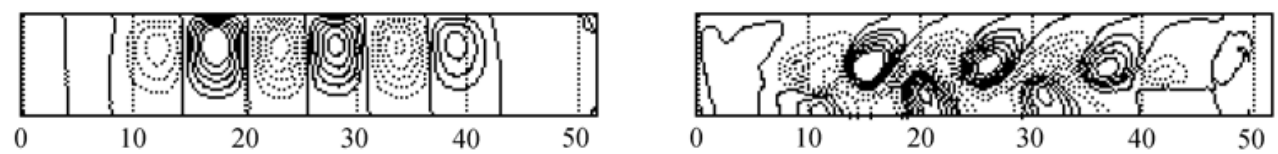

(a)
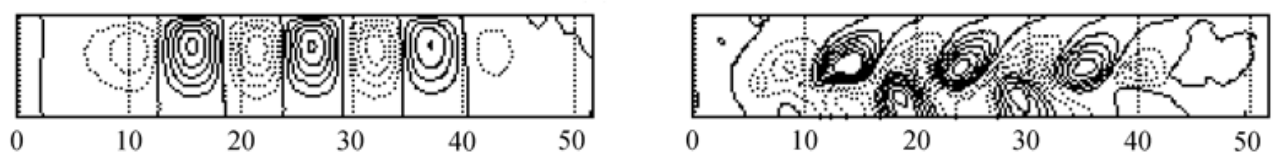

(b)
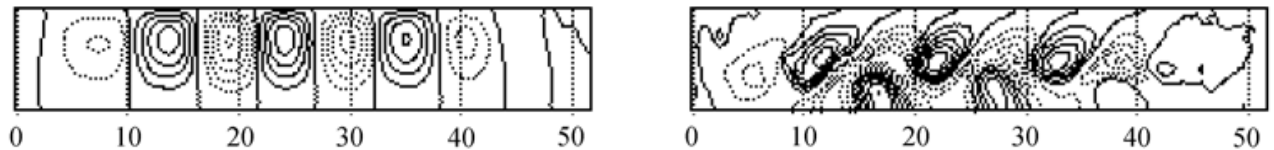

(c)
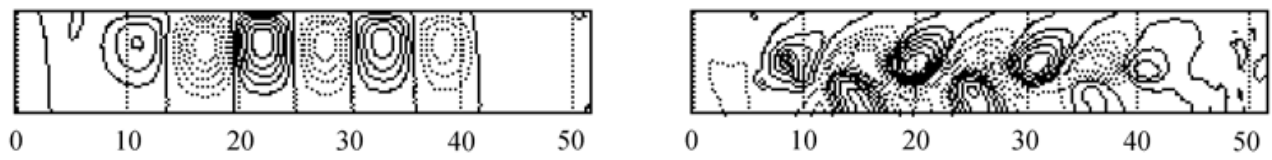

(d)

Figure 11 Stream lines (left) and isotherms (right) of the disturbed field in the liquid layer. $g=0, \Delta T=25^{\circ} \mathrm{C}$. (a) $t=600 \mathrm{~s}$; (b) $t=604 \mathrm{~s}$; (c) $t=608 \mathrm{~s}$; (d) $t=612 \mathrm{~s}$.
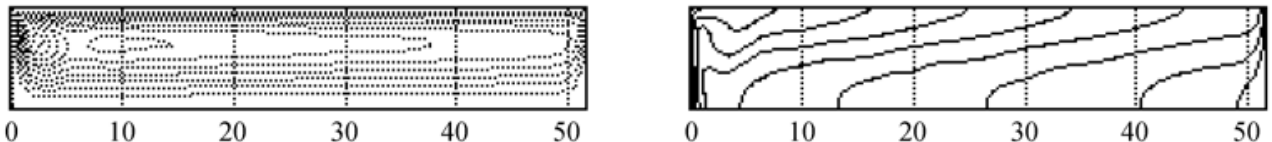

(a)
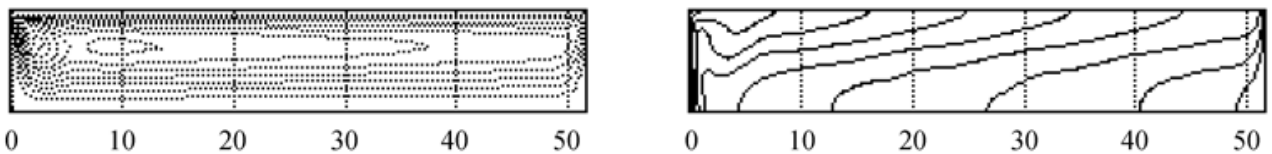

(b)
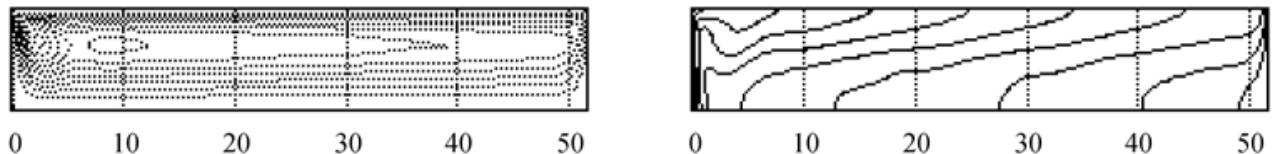

(c)
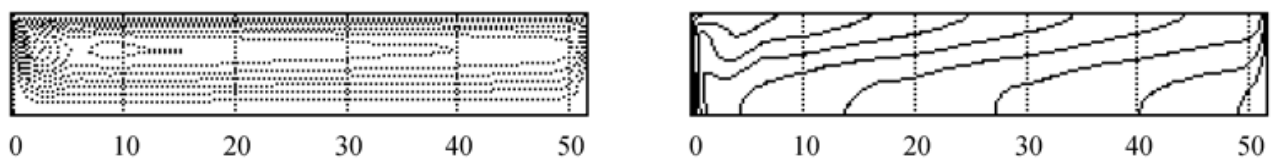

(d)

Figure 12 Temporary stream lines (left) and isotherms (right) in the liquid layer in the case of $g=0$ and $\Delta T=25^{\circ} \mathrm{C}$. (a) $t=600 \mathrm{~s}$; (b) $t=604 \mathrm{~s}$; (c) $t=608 \mathrm{~s}$; (d) $t=612 \mathrm{~s}$. 
Table 3 The magnitudes of $u^{\prime}, T^{\prime}$ and $P^{\prime}$ as well as the values of $u_{0 \max }, \Delta T$ and $P_{0 \max }\left(g=0, \Delta T=25^{\circ} \mathrm{C}\right)$

\begin{tabular}{ccccccccc}
\hline $\begin{array}{c}u_{\max }^{\prime} \\
\left(\mathrm{mm} \cdot \mathrm{s}^{-1}\right)\end{array}$ & $\begin{array}{c}u_{0 \max } \\
\left(\mathrm{mm} \cdot \mathrm{s}^{-1}\right)\end{array}$ & $\frac{u_{\max }^{\prime}}{u_{0 \max }}(\%)$ & $\begin{array}{c}T_{\max }^{\prime} \\
\left({ }^{\circ} \mathrm{C}\right)\end{array}$ & $\begin{array}{c}\Delta T_{0} \\
\left({ }^{\circ} \mathrm{C}\right)\end{array}$ & $\frac{T_{\max }^{\prime}}{\Delta T_{0}}(\%)$ & $\begin{array}{c}P_{\max }^{\prime} \\
\left(\mathrm{dyn} \cdot \mathrm{cm}^{-2}\right)\end{array}$ & $\begin{array}{c}P_{0 \max } \\
\left(\mathrm{dyn} \cdot \mathrm{cm}^{-2}\right)\end{array}$ & $\begin{array}{c}P_{\max }^{\prime} \\
P_{0 \max }\end{array}$ \\
\hline 20.0 & 3.9 & 0.51 & 0.012 & 25 & 0.05 & 0.00015 & 0.0391 \\
\hline
\end{tabular}

The phase relationship of the disturbed velocity $u^{\prime}$, temperature $T^{\prime}$ and pressure $P^{\prime}$ on the free surface is shown in Figure 13(a). The disturbed temperature and pressure have almost the same phase, which are $1 / 4$ period prior to the disturbed velocity $u^{\prime}$ in the direction of wave propagation. The maximum value of $T^{\prime}$ and $P^{\prime}$, the zero disturbed velocity $u^{\prime}$ in Figure 13(a) and the vertical zero-stream-function lines in Figure 13(b) are at the same places of the $x$ coordinate. The centers of the negative and positive $\psi^{\prime}$ cells correspond, respectively, to the maximum and minimum of $u^{\prime}$ (Figure 13(a)). We note that the disturbed velocity $u^{\prime}$ on the free surface moves from the higher disturbed temperature to the lower disturbed temperature $T^{\prime}$. These results demonstrate that the disturbance is driven by the thermal instability, and the wave observed is the hydrothermal wave.

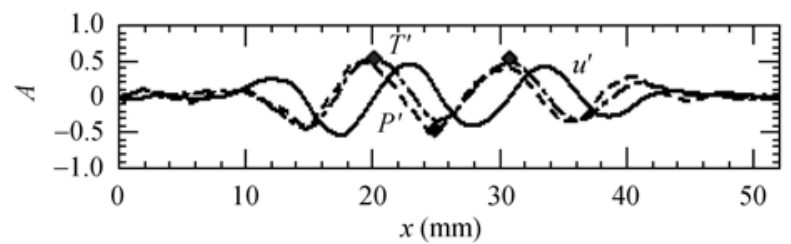

(a)

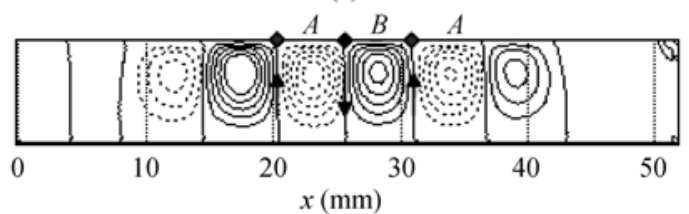

(b)

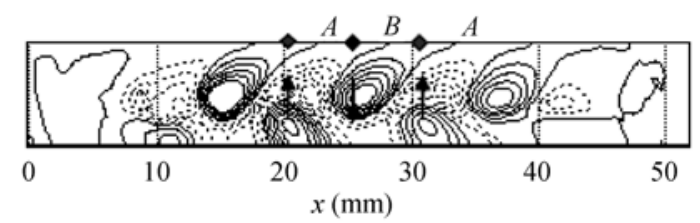

(c)

Figure 13 The temporal phase relationship of the disturbed velocity, temperature and pressure at $t=600 \mathrm{~s}$. (a) Disturbed velocity, temperature and pressure on the free surface; (b) disturbed stream function in the liquid layer ( - positive, ---- negative); (c) disturbed temperature in the liquid layer (— positive, ---- negative).

\section{Conclusions and discussion}

The traveling wave in the large- $P r$-number liquid layer is exited when the temperature difference between the left and right side walls of the cavity is high enough, and propagates towards the hot wall. The liquid layer of $4 \mathrm{~mm}$ high with the aspect ratio $l / h=13$ is studied in the case of $g=0$ in the present paper. When $\Delta T=25^{\circ} \mathrm{C}$, the disturbed cells extend to all regions of the liquid layer. The magnitude of the flow and temperature field in the liquid layer, the surface pressure and deformation of the free surface as well as the phase relationship of them are obtained. The disturbed surface temperature and disturbed pressure have almost the same phase, $1 / 4$ period prior to the disturbed velocity $u^{\prime}$ in the direction of wave propagation. The upstream traveling wave, named hydro- 
thermal wave, is driven by the surface tension gradient induced by the disturbed temperature. The magnitudes of disturbed temperature and flow fields are so small that the temporary total field and average field are almost the same. The temporal total field looks like a steady one. No oscillatory multi-cellular flow is observed from the total field. It is better to use the spectrum of flow or temperature at a point of the liquid layer to distinguish whether the hydrothermal wave appears in the small-size experiment on the ground or in the space. The surface oscillation can also be sensitively detected by the optical method.

The experiments of a half floating zone in space demonstrate that the thermocapillary convection becomes oscillatory if the applied temperature difference is over a critical value. Such a strong oscillatory convection can be observed directly from the total flow field in the experiment. It seems that the mechanism exciting the oscillation in the liquid bridge is different from that forming the hydrothermal wave in the liquid layer.

Both Benard convection (for $g \neq 0$ ) induced by buoyancy instability and Marangoni convection resulting from Pearson instability deal with the transition from the static to flow state in a liquid layer heated from the bottom. The two convections mentioned above cannot be induced in the liquid layer with the temperature gradient being positive along $z$-axis, as discussed in the present paper, and the liquid layer is stable in this meaning. The hydrothermal wave deals with the transition from steady to oscillatory thermocapillary convection in the liquid layer with a positive temperature gradient toward $z$-axis. In 1984 Smith and Davis ${ }^{[4]}$ pointed out that the velocity field is coupled with the temperature field through the thermocapillary shear-stress conditions on the interface. The mechanism for instability is associated with a balance between heat convection and heat conduction in the layer. The results of the present paper show the flow field, temperature and pressure distribution during the moment of the exciting hydrothermal wave. The temperature is transferred by average and the disturbed velocity. The disturbed isothermal cells are oblique to the free surface, as shown in Figure 13(c), where the disturbed temperature cells, expressed with the solid line, have the negative temperature gradient in the region lower than and near the free surface. At the hot disturbed temperature point on the free surface heat energy is obtained, which is supplied by the vertical disturbed velocity from the interior of the layer, thus inducing and maintaining the coupled action of Marangoni convection and thermocapillary convection. This results in the hydrothermal wave. The physical patterns and the phase relationship of velocity, temperature and pressure obtained in the present calculation are useful for understanding the mechanism exciting hydrothermal wave.

1 Schwabe D, Scharmann A, Preisser F, et al. Experiments on surface tension driven flow in floating zone melting. J Crystal Growth, 1978, 43: 305-312

2 Chun $\mathrm{Ch} \mathrm{H}$, Wuest W. Experiments on the transition from the steady to oscillatory Marangoni convection of a floating zone under reduced gravity effect. Acta Astron, 1979, 6: 1073-1082

3 Pearson J R A. On convection sells induced by surface tension. J Fluid Mech, 1958, 4: 489-500

4 Smith M K, Davis S H. Instability of dynamic thermocapillary liquid layer (I): Convective instability. J Fluid Mech, 1983, 132: 119-144[DOI]

5 Burguete J, Mukolobwiez N, Daviaud F, et al. Buoyant-thermocapillary instabilities in extended liquid layers subjected to a horizontal temperature gradient. Phys Fluids, 2001, 13: 2773-2787[DOI]

6 Villers D, Platten J K. Coupled buoyancy and Marangoni convection in acetone: experiments and comparison with numerical simulation. J Fluid Mech, 1992, 234: 487-510[DOI]

7 Shevtsova V M, Nepomnyashchy A A, Legros J C. Thermocapillary-buoyancy convection in a shallow cavity heated from the side. Phys Rev, 2003, 67: 066308-1-14

8 Carpenter B M, Homsy G M. High Marangoni number convection in a square cavity. Phys Fluids A, 1990, 2: 137$149[\mathrm{DOI}]$ 